\title{
La homeostasis de tiol/disulfuro es un indicador del estrés oxidativo en los niños críticamente enfermos con sepsis
} Thiol-disulphide homeostasis is an oxidative stress indicator in
critically ill children with sepsis

Dra. Ganime Ayar , Dra. Sanliay Sahin ${ }^{b}$ Dra. Yasemin Men Atmaca ${ }^{a}$, Dra. Mutlu Uysal Yazicia, Dr. Salim Neseliogluc y Dr. Ozcan Erelc

\section{RESUMEN}

Objetivo. Evaluar un novedoso marcador del estrés oxidativo (la homeostasis de tiol/ disulfuro) en la sepsis pediátrica y determinar sus efectos sobre el pronóstico de esta afección. Métodos. En el estudio, se incluyeron pacientes con diagnóstico de sepsis y controles sanos. Se midieron las concentraciones de tiol total, tiol nativo, disulfuro, disulfuro/ tiol total, disulfuro/ tiol nativo y tiol nativo/tiol total en los grupos con sepsis y de referencia. Se compararon los parámetros entre los supervivientes y los no supervivientes del grupo con sepsis. Se midieron las concentraciones de hemoglobina, leucocitos, trombocitos, lactato y proteína C-reactiva en los pacientes con sepsis al momento del diagnóstico. Se utilizaron el puntaje de riesgo de mortalidad pediátrico (Pediatric Risk of Mortality, PRISM) y el puntaje de disfunción orgánica (Pediatric Logistic Organ Dysfunction, PELOD) para estimar la gravedad de la enfermedad.

Resultados. En el grupo con sepsis se incluyó a 38 pacientes y en el de referencia, a 40 niños sanos. Las concentraciones plasmáticas de tiol en los pacientes con sepsis fueron significativamente inferiores que las del grupo de referencia $(p<0,001)$.

Conclusión. La homeostasis detiol/disulfuro fue anormal en los niños con sepsis en la unidad de cuidados intensivos pediátricos.

Palabras clave: sepsis, niño, estrés oxidativo, tioll disulfuro, homeostasis.

http: / / dx.doi.org/10.5546/ aap.2019.143

Texto completo en inglés:

http: / / dx.doi.org/10.5546/ aap.2019.eng.143

Cómo citar: Ayar G, Sahin S, Men Atmaca Y, Uysal Yazici M, et al. La homeostasis de tiol/disulfuro es un indicador del estrés oxidativo en los niños críticamente enfermos con sepsis. Arch Argent Pediatr 2019;117(3):143-148.

Correspondencia:

Dra. Ganime Ayar:

ganimeayar@gmail.com

Financiamiento:

Ninguno.

Conflicto de intereses:

Ninguno que declarar.

Recibido: $10-8-2018$

Aceptado: 12-12-2018

\section{INTRODUCCIÓN}

La sepsis es una de las principales causas de morbimortalidad en los lactantes y los niños. ${ }^{1,2}$ La tasa de mortalidad en los casos de sepsis pediátrica varía entre el $5 \%$ y el $40 \%$, a pesar de la detección y de la intervención agresiva tempranas en los pacientes con sepsis grave y choque séptico. ${ }^{3}$ Aproximadamente el $25 \%$ de las muertes en la unidad de cuidados intensivos pediátricos (UCIP) están relacionadas con infecciones graves, y las tasas de letalidad se elevan hasta el $17 \%{ }^{3}$ Recientemente se informó que está aumentando la incidencia de infecciones graves en los niños que requieren hospitalización en la UCIP. ${ }^{4-6}$

El equilibrio entre los oxidantes y los antioxidantes en el organismo es esencial para mantener las funciones bioquímica y celular. ${ }^{7} \mathrm{El}$ estrés oxidativo produce lesión celular cuando el nivel de especies reactivas del oxígeno aumenta por encima de los niveles fisiológicos en las células expuestas a los oxidantes endógenos o exógenos. ${ }^{8}$ Los oxidantes dañan los lípidos, las proteínas y el $\mathrm{ADN}$ a nivel celular, e incluso pueden causar muerte celular. En los pacientes que fallecieron debido a sepsis, las microscopías óptica y electrónica y la inmunohistoquímica para detectar marcadores de lesión celular y estrés revelaron que la muerte celular era rara en los casos de disfunción cardíaca y renal inducida por sepsis. Asimismo, el grado de lesión o muerte celular no representó la gravedad de la disfunción orgánica inducida por sepsis. ${ }^{9}$ 
El estrés oxidativo y los sistemas de defensa antioxidante también son factores que contribuyen a la patogenia de la sepsis. En los estudios, se notificó un aumento de la cantidad de especies reactivas del oxígeno producto del estrés oxidativo en los pacientes a los que se les diagnosticó sepsis. ${ }^{10}$ Se ha notificado que el potencial antioxidante podría ser bajo en los pacientes con sepsis al inicio de la enfermedad en comparación con los sujetos sanos, pero luego comienza a aumentar hasta valores normales y superiores. ${ }^{7,10}$

Los tioles plasmáticos son antioxidantes poderosos que eliminan los radicales libres fisiológicamente. Las concentraciones séricas de tioles proteicos son algunos de los indicadores del estado antioxidante del organismo. En general, existe un equilibrio entre los tioles y los disulfuros y también actúan como protectores de la homeostasis de oxidorreducción. Esta se denomina homeostasis dinámica de tiol/ disulfuro. Cuando la homeostasis de oxidorreducción se inclina hacia un desequilibrio de radicales libres, también se produce estrés oxidativo. Este proceso puede estar asociado con varias enfermedades. ${ }^{11,13}$

El objetivo de este estudio fue investigar la homeostasis de tiol/disulfuro en pacientes pediátricos con sepsis y explorar sus efectos en el pronóstico de la sepsis.

\section{MATERIALES Y MÉTODOS \\ Población del estudio}

Este estudio de casos y controles se llevó a cabo entre marzo de 2015 y febrero de 2016. Participaron en el estudio niños de entre 1 mes y 18 años de edad. El grupo del estudio estaba formado por niños con diagnóstico de sepsis y que recibían seguimiento en la UCIP del Hospital de Formación e Investigación en Salud Infantil y Enfermedades Pediátricas, Hematología y Oncología de la Universidad de Ciencias de la Salud, Angora, Turquía.

El grupo de referencia estaba compuesto por niños sanos de la misma edad y del mismo sexo. Para este, se seleccionó a niños sanos sin diagnóstico de una psicopatología ingresados a los consultorios pediátricos externos para controles de rutina.

En este estudio, se consideró que los pacientes tenían "sepsis" según lo establecido por el tercer consenso internacional para la definición de sepsis y shock séptico (Sepsis-3) ${ }^{14,15}$ con base en los resultados clínicos o de laboratorio. Estos criterios abarcan lo siguiente: 1 ) dos o más entre los siguientes: a. temperatura $>38^{\circ} \mathrm{C} \mathrm{o}<36{ }^{\circ} \mathrm{C}$, b. frecuencia cardíaca $>90 / \mathrm{min}$, c. frecuencia respiratoria $>20 / \mathrm{min}$ o $\mathrm{PaCO}_{2}<32 \mathrm{mmHg}$ $(4,3 \mathrm{kPa}) ; \mathrm{d}$. recuento de leucocitos $>12000 /$ $\mathrm{mm}^{3} \mathrm{o}<4000 / \mathrm{mm}^{3} \mathrm{o}>10 \%$ de células en bandas (neutrófilos inmaduros); 2) al menos uno de los siguientes criterios: hemocultivo positivo o sepsis clínica (cuando el hemocultivo era negativo).

Se excluyó a los pacientes con los siguientes criterios: edad $<3$ meses y $>18$ años, inmunosupresión, tratamiento con corticoesteroides o radioterapia, enfermedades crónicas (como diabetes mellitus, insuficiencia renal crónica), enfermedades reumatológicas, cáncer y el uso de un antioxidante (p. ej., vitaminas $\mathrm{E}$ y C). Se incluyó en el estudio a los pacientes que aceptaron participar y cumplieron con los criterios de inclusión. El Comité de Ética local aprobó el estudio. Se obtuvo el consentimiento informado de los padres o cuidadores de los pacientes.

\section{Procedimientos}

Se midieron los valores de hemoglobina, leucocitos, trombocitos, lactato, proteínas totales y proteína C-reactiva (PCR) en las muestras de sangre obtenidas de los pacientes con sepsis al momento del diagnóstico o en un plazo de 72 horas después de este.

Se utilizaron el puntaje de riesgo de mortalidad pediátrico (Pediatric Risk of Mortality, PRISM III) y el puntaje de disfunción orgánica (Pediatric Logistic Organ Dysfunction, PELOD) para estimar la gravedad de la enfermedad.

Se recolectaron las muestras de sangre en ayunas de los grupos de pacientes y de referencia en tubos lisos (BD Vacutainer ${ }^{\circledR}$, SST II Advance, REF 367955, Reino Unido) para medir los antioxidantes. Se centrifugaron las muestras de sangre a $1500 \mathrm{~g}$ durante 10 minutos en el transcurso de 20 minutos posteriores a la recolección para separar las muestras de suero, y luego se las conservó a $-80{ }^{\circ} \mathrm{C}$ hasta el momento del análisis.

\section{Mediciones}

\section{Método de laboratorio}

Se determinaron las concentraciones de tiol total, tiol nativo, disulfuro, disulfuro/tiol total, disulfuro/tiol nativo y tiol nativo/tiol total.

Las concentraciones de tiol total y tiol nativo en las muestras se midieron con el reactivo de Ellman y el reactivo de Ellman modificado. El contenido de tiol nativo se restó del contenido de 
tiol total y se calculó la mitad de esta diferencia; este valor era una cantidad de enlaces disulfuro dinámicos. Se calcularon las proporciones de disulfuro/tiol total, disulfuro/tiol nativo y tiol nativo/tiol total.

La prueba de la homeostasis de tiol/disulfuro se realizó mediante espectrofotometría según lo describen Erel y col., ${ }^{13}$ Se utilizaron kits caseros en el analizador automático Cobas C501 (RocheHitachi, Alemania) conforme al método descripto.

\section{Análisis estadístico}

Se empleó la prueba de Shapiro-Wilk para verificar si las variables continuas del estudio (edad, proteínas totales y tioles totales) tenían una distribución normal. Las estadísticas descriptivas se expresaron en números y porcentajes para las variables categóricas y en media, desviación estándar y mediana (intervalo intercuartílico, IIC) para las variables numéricas. Las variables con distribución normal se expresaron como media y desviación estándar $(\mathrm{M} \pm \mathrm{DE})$; aquellas con una distribución anormal, como mediana e IIC (percentilo 25-75); y las variables categóricas (p. ej., sexo), como porcentajes (\%). Se empleó la prueba U de Mann-Whitney para comparar las variables numéricas con distribución anormal entre ambos grupos. La prueba $t$ de Student se usó para comparar las variables numéricas con distribución normal entre ambos grupos. El nivel de significación estadística se estableció en $p<0,05$. Se usó el programa informático SPSS

TABLA 1. Distribución de la edad y el sexo en los grupos con sepsis y de referencia

\begin{tabular}{lccc}
\hline & Grupo con sepsis $(\mathbf{n}=\mathbf{3 8})$ & Grupo de referencia $(\mathbf{n}=4 \mathbf{4 0})$ & Valor $\boldsymbol{p}$ \\
\hline Sexo, $\mathbf{n}(\%)$ & $17(51,4)$ & $20(50,0)$ & 0,827 \\
$\quad$ Femenino & $21(48,6)$ & $20(50,0)$ & 0,408 \\
$\quad$ Masculino & $3,75(1,375-11,575)$ & $4,75(2,250-9,875)$ & \\
Edad, años, mediana (IIC) &
\end{tabular}

IIC: intervalo intercuartílico.

TABLA 2. Concentraciones de antioxidantes en los grupos con sepsis y de referencia

\begin{tabular}{|c|c|c|c|}
\hline & $\begin{array}{l}\text { Grupo con sepsis } \\
(\mathrm{n}=38)\end{array}$ & $\begin{array}{c}\text { Grupo de referencia } \\
(n=40)\end{array}$ & Valor $p$ \\
\hline & Mediana (IIC) & Mediana (IIC) & \\
\hline Tiol nativo $(\mu \mathrm{mol} / \mathrm{L})$ & $260,75(220,9-332,0)$ & $451,40(431,93-463,95)$ & $<0,001$ \\
\hline Tiol total $(\mu \mathrm{mol} / \mathrm{L})$ & $284,25(241,53-361,15)$ & $483,85(465,98-504,33)$ & $<0,001$ \\
\hline Disulfuro $(\mu \mathrm{mol} / \mathrm{L})$ & $13,48(10,24-17,63)$ & $19,43(15,26-21,64)$ & $<0,001$ \\
\hline Disulfuro/tiol nativo & $5,25(4,00-6,24)$ & $4,14(3,27-4,80)$ & 0,004 \\
\hline Disulfuro/tiol total & $4,75(3,59-5,55)$ & $3,82(3,13-4,38)$ & 0,006 \\
\hline Tiol nativo/tiol total & $90,35(88,14-91,96)$ & $92,32(91,23-93,75)$ & 0,001 \\
\hline
\end{tabular}

IIC: intervalo intercuartílico.

TABLA 3. Características clínicas y de laboratorio de los supervivientes y los no supervivientes en el grupo con sepsis

\begin{tabular}{|c|c|c|c|}
\hline & $\begin{array}{l}\text { Supervivientes } \\
\quad(n=27)\end{array}$ & $\begin{array}{c}\text { No supervivientes } \\
(\mathrm{n}=11)\end{array}$ & Valor $p$ \\
\hline Sexo & $\mathrm{n}(\%)$ & $\mathrm{n}(\%)$ & \\
\hline Femenino & $14(52)$ & $4(36)$ & 0,392 \\
\hline \multirow[t]{2}{*}{ Masculino } & $13(48)$ & $7(64)$ & \\
\hline & Mediana (IIC) & Mediana (IIC) & \\
\hline Edad, años & $4,0(1,5-11,5)$ & $2,5(1,0-11,8)$ & 0,628 \\
\hline Leucocitos, $x 103 / \mu 1$ & $12,2(9,0-21,3)$ & $7,8(3,5-24,3)$ & 0,664 \\
\hline Hemoglobina, g/dl & $10,9(9,0-11,8)$ & $10,6(9,2-13,0)$ & 0,923 \\
\hline Trombocitos, x103/ $\mu 1$ & $193,0(51,0-315,0)$ & $136,0(24,0-367,0)$ & 0,987 \\
\hline $\mathrm{PCR}, \mathrm{mg} / \mathrm{dl}$ & $7,8(1,7-19,0)$ & $14,2(2,9-20,9)$ & 0,552 \\
\hline Lactato, $\mathrm{mmol} / \mathrm{l}$ & $2,1(1,4-3,9)$ & $2,7(1,7-4,2)$ & 0,384 \\
\hline \multirow[t]{2}{*}{ Proteínas totales, g/dl } & $5,6(5,2-6,1)$ & $5,9(5,3-6,2)$ & 0,519 \\
\hline & Media \pm DE & Media \pm DE & \\
\hline PRISM III, puntaje & $14,89 \pm 9,39$ & $21,55 \pm 10,91$ & 0,067 \\
\hline PELOD, puntaje & $18,96 \pm 13,02$ & $29,27 \pm 15,81$ & $<0,05$ \\
\hline
\end{tabular}

PCR: proteína C-reactiva; IIC: intervalo intercuartílico; DE: desviación estándar. 
Statistics de IBM para Windows, versión 21.0 (IBM Corp., lanzado en 2012, Armonk, NY) para los análisis y cálculos estadísticos.

\section{RESULTADOS}

En la Figura 1, se muestra el diagrama de flujo de los pacientes. En el grupo con sepsis se incluyó a 38 pacientes (17 niñas/21 niños), cuya mediana de edad era de 3,75 años. En el grupo de referencia se incluyó a 40 niños sanos (20 niñas / 20 niños), cuya mediana de edad era de 4,75 años. No se observó ninguna diferencia estadísticamente significativa en cuanto a la edad y al sexo entre el grupo con sepsis y el grupo de referencia. Las características demográficas de los pacientes se describen en la Tabla 1.

En la Tabla 2, se muestra la distribución de tiol nativo, tiol total y disulfuro por grupo. Las concentraciones de tiol nativo, tiol total y disulfuro fueron significativamente más bajas $(p<0,001)$ en los pacientes con sepsis en comparación con el grupo de referencia. La proporción de disulfuro/tiol nativo y de disulfuro/tiol total fue más elevada $(p<0,05)$ en los pacientes con sepsis.

La tasa de mortalidad de los pacientes con diagnóstico de sepsis fue del 28,9\% (11 de 38). Entre los pacientes con sepsis, no se observaron diferencias significativas entre los supervivientes y los no supervivientes en relación con la edad, el sexo y los valores críticos de laboratorio (Tabla 3 ).

En la Tabla 4, se comparan los parámetros de la homeostasis de tiol / disulfuro al momento del diagnóstico de sepsis entre los supervivientes $(\mathrm{n}=27)$ y los no supervivientes $(\mathrm{n}=11)$. No se observaron diferencias significativas en los valores de tiol.

No se determinó una correlación estadísticamente significativa entre los parámetros de la homeostasis de tiol/disulfuro y los puntajes PRISM y PELOD (no se muestran los datos).

\section{DISCUSIÓN}

La patogenia de la sepsis constituye una situación bastante compleja en la que participan numerosos mediadores y diversos biomarcadores. La función de los antioxidantes en la sepsis se basa en una cantidad limitada de estudios realizados en adultos. ${ }^{8,9} \mathrm{Si}$ bien se notificó previamente el uso de las concentraciones de tioles como parámetro antioxidante en otras enfermedades, ${ }^{16-18}$ este es el primer estudio que investigó la homeostasis dinámica de tiol/disulfuro en niños con sepsis. La homeostasis dinámica de tiol/disulfuro tiene una función esencial en la protección antioxidante como marcador novedoso del estrés oxidativo que, cada vez más, está implicado en muchos

FIgURA 1. Diagrama de flujo de los pacientes

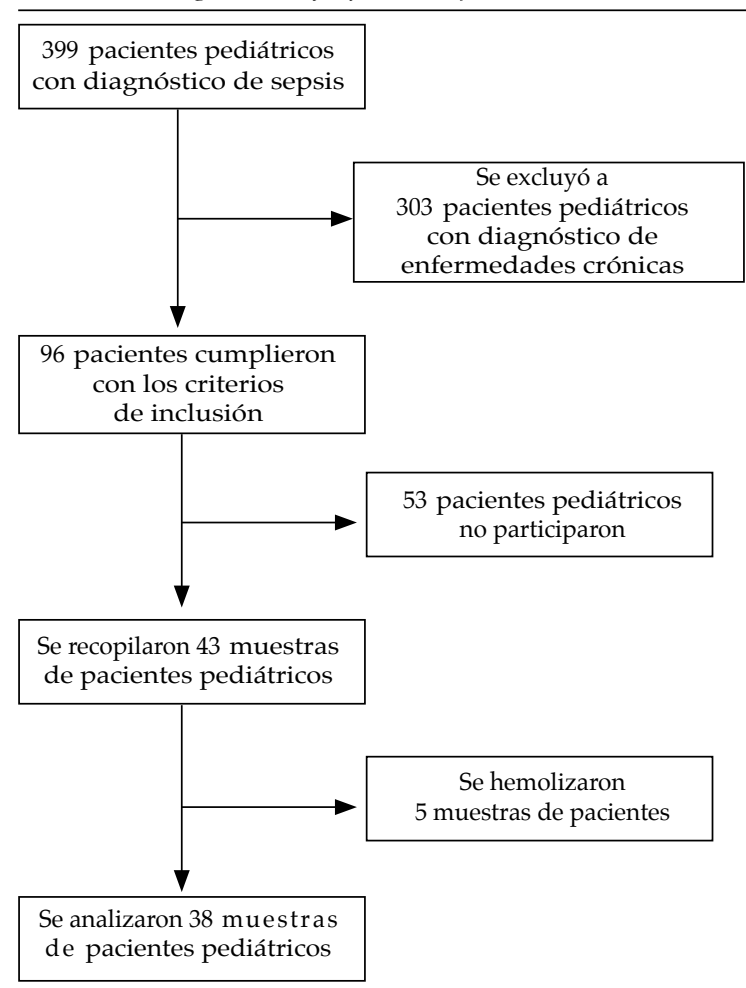

Tabla 4. Parámetros de la homeostasis de tiol/disulfuro en los supervivientes y los no supervivientes del grupo con sepsis

\begin{tabular}{lccc}
\hline Variable & Supervivientes $(\mathbf{n}=\mathbf{2 7})$ & No supervivientes $(\mathbf{n}=\mathbf{1 1})$ & Valor $\boldsymbol{p}$ \\
\hline Tiol nativo $(\mu \mathrm{mol} / \mathbf{l})$ & $268,4(197,7-332,9)$ & $249,9(230,0-342,2)$ & 0,834 \\
Tiol total $(\mu \mathrm{mol} / \mathbf{l})$ & $296,4(223,3-358,1)$ & $275,5(254,8-371,0)$ & 0,961 \\
Disulfuro $(\mu \mathrm{mol} / \mathbf{l})$ & $14,2(10,2-19,1)$ & $12,2(11,0-17,5)$ & 0,489 \\
Disulfuro/tiol nativo & $5,4(4,1-6,7)$ & $5,1(3,1-5,6)$ & 0,253 \\
Disulfuro/tiol total & $4,9(3,7-5,9)$ & $4,7(3,0-5,0)$ & 0,296 \\
Tiol nativo/tiol total & $89,8(87,8-91,9)$ & $90,7(89,9-94,1)$ & 0,119 \\
\hline
\end{tabular}

Los parámetros se expresan como mediana e intervalo intercuartílico. 
trastornos; su determinación brinda información valiosa sobre varios procesos bioquímicos normales o anormales. ${ }^{11}$

Observamos que los valores de tiol total, tiol nativo y disulfuro, y la proporción de tiol nativo/tiol total fueron inferiores en los pacientes pediátricos con sepsis. Esto indica que el equilibrio de antioxidantes se ve afectado en los pacientes con sepsis. Estos resultados fueron similares a los informados en relación con la capacidad antioxidante, estudiada previamente en pacientes con sepsis y críticamente enfermos. ${ }^{19-21}$ En nuestro estudio, la proporción de disulfuro/ tiol nativo y de disulfuro/tiol total fue más elevada en los pacientes con sepsis. Esto lo atribuimos a una mayor reducción de tiol nativo y de tiol total en comparación con la concentración de disulfuro.

Si bien la cantidad de estudios en pacientes pediátricos es limitada, se estudió la actividad de las enzimas antioxidantes en algunos estudios realizados en adultos. Kumar y col., ${ }^{19}$ determinaron que los pacientes adultos con sepsis tenían concentraciones elevadas de oxidantes. Asimismo, también determinaron que las enzimas antioxidantes disminuyen en los casos de sepsis y, además, observaron una relación inversa entre las enzimas antioxidantes y las insuficiencias orgánicas.

En un estudio multicéntrico realizado por Lorente y col., ${ }^{20}$ se midió la capacidad antioxidante en pacientes con sepsis y se la comparó entre los supervivientes y los no supervivientes. Los autores establecieron una asociación entre la capacidad antioxidante total y la mortalidad en los pacientes con sepsis grave y determinaron que la capacidad antioxidante era un indicador capaz de predecir la evolución de la sepsis grave. En un estudio reciente, ${ }^{21}$ se examinó la capacidad antioxidante durante la primera semana en los pacientes adultos con sepsis y se estableció una asociación con la gravedad de la sepsis y la mortalidad. Sin embargo, Karapetsa y col. ${ }^{22}$ estudiaron la capacidad antioxidante total el primer día en los pacientes con choque séptico -que es un indicador de sepsis grave-, pero no observaron una diferencia significativa entre los supervivientes y los no supervivientes en cuanto al equilibrio de antioxidantes. De manera similar a lo observado en la bibliografía, el puntaje PELOD, un indicador de disfunción multiorgánica, fue alto en nuestros pacientes no supervivientes. ${ }^{20,21}$

Se sabe que el equilibrio antioxidante es importante en los pacientes con sepsis, en especial en aquellos con sepsis grave y disfunción multiorgánica alarmante. Se propuso que la disfunción orgánica inducida por sepsis se produce, en parte, debido a la disfunción mitocondrial causada por el estrés oxidativo $y$, a su vez, este produce insuficiencia energética. ${ }^{23}$

En nuestro estudio, analizamos la homeostasis de tiol/disulfuro en los pacientes pediátricos mediante un método novedoso y observamos que era significativamente inferior en los pacientes con sepsis en comparación con los controles.

Las limitaciones de este estudio son la cantidad pequeña de casos, la medición de las concentraciones de antioxidantes en una sola muestra de sangre y la realización en un solo centro. Dado que podrían ocurrir cambios en las concentraciones de antioxidantes según la evolución y el tratamiento de la enfermedad, las mediciones repetidas en muestras de sangre durante el seguimiento de los pacientes brindarían más información. Además, en nuestro estudio solo determinamos la homeostasis dinámica de tiol/disulfuro, que es simplemente una parte del complejo mecanismo del estrés oxidativo. No fue posible determinar las correlaciones entre la homeostasis dinámica de tiol/disulfuro y otros parámetros oxidativos.

\section{CONCLUSIONES}

La homeostasis dinámica de tiol/disulfuro se vio alterada en los pacientes con sepsis y esta es importante en los niños críticamente enfermos. Este método novedoso podría convertirse en una herramienta sencilla que podría aplicarse en los análisis de detección de rutina como indicador para demostrar el estrés oxidativo específico de los tioles, en especial en los casos de sepsis pediátrica. Sin embargo, se requieren estudios futuros que respalden estos hallazgos.

\section{REFERENCIAS}

1. Randolph AG, McCulloh RJ. Pediatric sepsis: important considerations for diagnosing and managing severe infections in infants, children, and adolescents. Virulence. 2014; 5(1):179-89.

2. WheelerDS, Wong HR. Sepsis in Pediatric Cardiac Intensive Care. Pediatr Crit Care Med. 2016; 17(8 Suppl 1):S266-71.

3. de Souza DC, Barreira ER, Faria LS. The Epidemiology of Sepsis in Childhood. Shock. 2017; 47(Suppl 1):2-5.

4. Schlapbach LJ, Straney L, Alexander J, MacLaren G, et al. Mortality related to invasive infections, sepsis, and septic shock in critically ill children in Australia and New Zealand, 2002-13: a multi centre retrospective cohort study. Lancet Infect Dis. 2015; 15(1):46-54.

5. Hartman ME, Linde-Zwirble WT, Angus DC, Watson RS. Trends in the epidemiology of pediatric severe sepsis. Pediatr Crit Care Med. 2013; 14(7):686-93. 
6. Balamuth F, Weiss SL, Neuman MI, Scott H, et al. Pediatric severe sepsis in U.S. children's hospitals. Pediatr Crit Care Med. 2014; 15(9):798-805.

7. Lorente L, Martín MM, Pérez-Cejas A, Abreu-González $\mathrm{P}$, et al. Serum total antioxidant capacity during the first week of sepsis and mortality. J Crit Care. 2018; 47:139-44.

8. Harrois A, Huet O, Duranteau J. Alterations of mitochondrial function in sepsis and critical illness. Curr Opin Anaesthesiol. 2009; 22(2):143-9.

9. Takasu O, Gaut JP, Watanabe E, To K, et al. Mechanisms of cardiac and renal dysfunction in patients dying of sepsis. Am J Respir Crit Care Med. 2013; 187(5):509-17.

10. Kumar S, Gupta E, Kaushik S, Kumar Srivastava V, Mehta SK, Jyoti A. Evaluation of oxidative stress and antioxidant status: Correlation with the severity of sepsis. Scand J Immunol. 2018; 87(4):e12653.

11. Davies KJ. Oxidative stress, antioxidant defenses, and damage removal, repair, and replacement systems. IUBMB Life. 2000; 50(4-5):279-89.

12. Haddad JJ. Oxygen sensing and oxidant/redox-related pathways. Biochem Biophys Res Commun. 2004;316(4):969-77.

13. ErelO, NeseliogluS. A novel and automated assay for thiol/ disulphide homeostasis. Clin Biochem. 2014; 47(18):326-32.

14. Singer M, Deutschman CS, Seymour CW, Shankar-HariM, et al. The Third International Consensus Definitions for Sepsis and Septic Shock (Sepsis-3). JAMA. 2016; 315(8):801-10.

15. Goldstein B, Giroir B, Randolph A; International Consensus Conference on Pediatric Sepsis. International pediatric sepsis consensus conference: definitions for sepsis and organ dysfunction in pediatrics. Pediatr Crit Care Med. 2005; 6(1):2-8
16. Ozler S, Oztas E, Caglar AT, Uygur D, et al. Thiol/disulfide homeostasis in predicting adverse perinatal outcomes at 24-28 weeks of pregnancy in gestational diabetes. J Matern Fetal Neonatal Med. 2016; 29(22):3699-704.

17. Kundi H, Ates I, Kiziltunc E, Cetin M, et al. A novel oxidative stress marker in acute myocardial infarction; thiol/disulphide homeostasis. Am J Emerg Med. 2015; 33(11):1567-71.

18. Ates I, Ozkayar N, Inan B, Yilmaz FM, et al. Dynamic thiol/disulphide homeostasis in patients with newly diagnosed primary hypertension. J Am Soc Hypertens. 2016; 10(2):159-66.

19. Kumar S, Gupta E, Kaushik S, Kumar Srivastava V, et al. Evaluation of oxidative stress and antioxidant status: Correlation with the severity of sepsis. Scand J Immunol. 2018; 87(4):e12653.

20. Lorente L, Martín MM, Almeida T, Abreu-González P, et al. Association between serum total antioxidant capacity and mortality in severe septic patients. J Crit Care. 2015; 30(1):217.e7-12.

21. Lorente L, Martín MM, Pérez-Cejas A, Abreu-González $\mathrm{P}$, et al. Serum total antioxidant capacity during the first week of sepsis and mortality. J Crit Care. 2018; 47:139-44.

22. Karapetsa M, Pitsika M, Goutzourelas N, Stagos D, et al. Oxidative status in ICU patients with septic shock. Food Chem Toxicol. 2013; 61:106-11.

23. Galley HF. Oxidative stress and mitochondrial dysfunction in sepsis. Br J Anaesth. 2011; 107(1):57-64.

\section{"Educación, no es llenar el cubo, sino encender el fuego".}

